MATHEMATICS OF COMPUTATION

Volume 70, Number 236, Pages 1397-1416

S 0025-5718(00)01263-1

Article electronically published on July 21,2000

\title{
STABILIZED WAVELET APPROXIMATIONS OF THE STOKES PROBLEM
}

\author{
CLAUDIO CANUTO AND ROLAND MASSON
}

\begin{abstract}
We propose a new consistent, residual-based stabilization of the Stokes problem. The stabilizing term involves a pseudo-differential operator, defined via a wavelet expansion of the test pressures. This yields control on the full $L^{2}$-norm of the resulting approximate pressure independently of any discretization parameter. The method is particularly well suited for being applied within an adaptive discretization strategy. We detail the realization of the stabilizing term through biorthogonal spline wavelets, and we provide some numerical results.
\end{abstract}

\section{INTRODUCTION}

Wavelet bases are being increasingly used in the numerical solution of partial differential and integral equations (see, e.g., [Da2, $\mathrm{Co}$, and the references therein). There are many aspects in a discretization procedure for such equations that can benefit from the features of these bases. Wavelets share with other multilevel methods the capability of easily preconditioning the discrete realizations of symmetric positive definite operators. More typical of wavelets is their orthogonality to certain classes of smooth functions (e.g., polynomials), a feature which can be exploited in the compression of dense matrices and - in a more general context - in the design of adaptive discretization strategies. The finite-dimensional space, which is used in a Galerkin-type approximation, is adaptively constructed by including in it precisely those wavelet basis functions that have the potential of representing the most significant structures of the solution. From this point of view, wavelet projection methods can be viewed as meshless methods, with a highly flexible mechanism for adding/removing degrees of freedom.

Wavelets were originally introduced in unbounded domains, with a shift invariant property (see $[\mathrm{Me}]$ ). Currently, wavelet bases are available and easily computable on fairly general domains in an arbitrary dimension. A popular strategy of construction consists of decomposing the domain into the union of smooth images of a tensor product reference domain. The wavelets are themselves images of tensor product wavelets on such a reference domain; this allows the by now well-developed wavelet

Received by the editor June 4, 1999 and, in revised form, October 18, 1999.

2000 Mathematics Subject Classification. Primary 65N30, 65N12, 42C15.

Key words and phrases. Stokes problem, inf-sup condition, stabilization, wavelet bases.

This work was partially supported by the European Commission within the TMR project (Training and Mobility for Researchers) Wavelets and Multiscale Methods in Numerical Analysis and Simulation, No. ERB FMRX CT98 0184, and by the Italian funds Murst 40\% Analisi Numerica. 
technology on the unit interval to be efficiently exploited (see [DS, CTU, CM2, BCU]).

Fluid dynamics is a challenging field of application for wavelet-based adaptive discretization methods, since typically a flow exhibits well-localized structures and/or coherent vortex patterns. For incompressible flows, the Stokes problem is a simplified model which neglects convection and focusses on the viscous effects and the divergence-free constraint. For these reasons, it has received considerable attention by wavelet addicts, beginning with the pioneering work of Lemarié $[\mathbf{L}$, in which divergence-free wavelet bases were constructed in an unbounded domain (see also [U]). However, for arbitrary bounded domains and boundary conditions, divergence-free basis functions are difficult to build; hence, one restorts to the discretization of the continuity equation, thus coping with the well-known inf-sup condition (see $[\mathrm{BF}]$ ) which ties together discrete velocity and pressure spaces. Several wavelet discretization methods have been recently proposed which fulfill that condition (see [DKU1, Ma2]). This task is relatively easy in the case of nonadaptive approximations, when the discrete velocity and pressure spaces are uniform, i.e., all the wavelet basis functions up to a certain maximal level are included. The situation becomes considerably more intriguing in the adaptive case, when the need for fulfilling the inf-sup condition contrasts against the desire to choose the discrete velocity and pressure spaces as independently as possible, guided only by the local structure of the flow.

Instead of satisfying the inf-sup condition, one can circumvent it. This alternative approach, first proposed by Hughes, Franca and Balestra [HFB] and now popular in the finite-element community (see, e.g., $[\overline{\mathrm{BF}}]$ ), can be realized by appending a suitable consistent (i.e., vanishing for the exact solution) stabilization term to the continuity equation. It prevents the onset of spurious oscillations in discrete pressure, making the approximate problem well posed. The same effect can be achieved by adding and then statically condensing auxiliary velocity functions, the "bubbles" (see [BFHR]). The typical stabilization term acts at the elemental level, and, through the choice of local tuning parameters, it provides stabilization by controlling a mesh-dependent weighted norm of the pressure gradient.

Among the features of wavelets (as well as other multilevel bases, see, e.g., $[\mathrm{BP}$ ), we recall the possibility of easily representing norms and inner products in Sobolev spaces of fractional and even negative order. This can be exploited to design new stabilized discretizations of operator equations. Such formulations are optimal from the point of view of the functional setting. Some results on the use of wavelets in this direction already exist. In $\mathrm{B}$, a multilevel least-square stabilization of the Stokes problem is considered, whereas in [BCT] a multilevel SUPG-type stabilization of the convection-diffusion equation yields control on some norm of fractional order $1 / 2$ for the solution.

In the present paper, we exploit wavelets to design a new, consistent, residualbased stabilization term for the Stokes problem, which replaces the classical term introduced in [HFB] for finite elements. Our term yields direct control of the full $L^{2}$ norm of the pressure, independently of any discretization parameter. Furthermore, basically no information on the discrete velocity space is needed. Consequently, the method is particularly well suited for the discretization of the problem in an adaptive framework as described above. Technically speaking, our term exploits the expansion of the discrete pressures in a wavelet basis (associated with a possibly nonconforming decomposition of the domain into macroelements), and the existence of a local dual basis. A local right-inverse of the divergence operator is easily built 
on the space spanned by the latter basis. This operator is used to define the test functions for the residual of the momentum equation, thus yielding the stabilization device.

The content of the paper is as follows. First, we introduce our stabilization term in an abstract setting, and we prove that it implies a uniform inf-sup condition for both the continuous and the discrete velocity-pressure pairs of spaces. This yields optimal a priori and a posteriori error estimates. Next, we detail a particular construction of the stabilizing wavelets, based on the Cohen-Daubechies-Feauveau CDF biorthogonal spline wavelets on the real line. Finally, we describe the results of some numerical tests which demonstrate the feasibility of the proposed method.

The following notation will be used throughout the paper. If, for $i=1,2$, $N_{i}$ are nonnegative functions defined on sets $A_{i}$ which may depend on certain parameters, then $N_{1}\left(a_{1}\right) \lesssim N_{2}\left(a_{2}\right)$ means the existence of a constant $c$ independent of these parameters such that $N_{1}\left(a_{1}\right) \leq c N_{2}\left(a_{2}\right), \forall a_{1} \in A_{1}, \forall a_{2} \in A_{2}$. Moreover, $N_{1}\left(a_{1}\right) \equiv N_{2}\left(a_{2}\right)$ means $N_{1}\left(a_{1}\right) \lesssim N_{2}\left(a_{2}\right)$ and $N_{2}\left(a_{2}\right) \lesssim N_{1}\left(a_{1}\right)$.

\section{An ABstract Form of the StABilization METHOD}

Given a bounded domain $\Omega \subset \mathbb{R}^{d}(d \geq 2)$ with Lipschitz boundary $\partial \Omega$, we want to approximate the Stokes problem submitted to homogeneous boundary conditions:

$$
\begin{aligned}
-\Delta \vec{U}+\nabla P & =\vec{f} & & \text { in } \Omega, \\
\nabla \cdot \vec{U} & =0 & & \text { in } \Omega, \\
\vec{U} & =0 & & \text { on } \partial \Omega .
\end{aligned}
$$

Let us introduce the function spaces $\vec{X}=\left(H_{0}^{1}(\Omega)\right)^{d}$ for velocities and $M \simeq L^{2}(\Omega) / \mathbb{R}$ (so that $\left.L^{2}(\Omega)=M \oplus \operatorname{span}(1)\right)$ for pressures. Existence and uniqueness of a solution $(\vec{U}, P) \in \vec{X} \times M$ follow classically from the assumption $\vec{f} \in \vec{X}^{\prime}=\left(H^{-1}(\Omega)\right)^{d}$. Throughout the paper, both the $\left(L^{2}(\Omega)\right)^{d}$-inner product and the $M$-inner product will be denoted by $(\cdot, \cdot)$; the symbol $\langle\cdot, \cdot\rangle$ will indicate the duality pairing between $\vec{X}^{\prime}$ and $\vec{X}$. Let us equip $\vec{X}$ by the norm $\|\vec{v}\|_{\vec{X}}=(\nabla \vec{v}, \nabla \vec{v})^{1 / 2}$ and $M$ by the norm $\|q\|_{M}=(q, q)^{1 / 2}$.

In order to define the stabilizing operator and the approximation spaces, let us assume that $M$ is split as follows:

$$
M=M_{0} \oplus \mathcal{M},
$$

where the complementary spaces $M_{0}$ and $\mathcal{M}$ are closed subspaces of $M$ and satisfy the following conditions:

i) $\mathcal{M}$ admits a Riesz basis $\Psi^{p}=\left\{\psi_{\lambda}^{p}: \lambda \in \mathcal{L}^{p}\right\}$, i.e., $\mathcal{M}=\operatorname{clos} \operatorname{span} \Psi^{p}$ with

$$
\left\|\sum_{\lambda \in \mathcal{L}^{p}} q_{\lambda} \psi_{\lambda}^{p}\right\|_{M} \sim\|\underline{q}\|_{\ell^{2}\left(\mathcal{L}^{p}\right)}
$$

for all $\underline{q}=\left(q_{\lambda}\right)_{\lambda \in \mathcal{L}^{p}} \in \ell^{2}\left(\mathcal{L}^{p}\right)$;

ii) $M_{0}$ is a (possibly empty) finite-dimensional subspace of $M$; if $M_{0} \neq \emptyset$, then a finite-dimensional subspace $\vec{X}_{0}$ of $\vec{X}$ is associated to it, such that the uniform inf-sup condition

$$
\exists \beta_{0}>0: \quad \inf _{q \in M_{0}} \sup _{\vec{v} \in \vec{X}_{0}} \frac{(\nabla \cdot \vec{v}, q)}{\|\vec{v}\|_{\vec{X}}\|q\|_{M}} \geq \beta_{0}
$$

holds. 
As a consequence of i) and the Riesz representation theorem, there exists a dual biorthogonal set $\tilde{\Psi}^{p}=\left\{\tilde{\psi}_{\lambda}^{p}: \lambda \in \mathcal{L}^{p}\right\} \subset M_{0}^{\perp}$; thus,

$$
\left(\psi_{\lambda}^{p}, \tilde{\psi}_{\lambda^{\prime}}^{p}\right)=\delta_{\lambda, \lambda^{\prime}}, \quad \forall \lambda, \lambda^{\prime} \in \mathcal{L}^{p}
$$

and any $q \in \mathcal{M}$ can be represented as

$$
q=\sum_{\lambda \in \mathcal{L}^{p}} q_{\lambda} \psi_{\lambda}^{p} \quad \text { with } \quad q_{\lambda}=\left(q, \tilde{\psi}_{\lambda^{\prime}}^{p}\right)
$$

Next, we associate a function $\vec{\psi}_{\lambda}^{*} \in \vec{X}$ and a coefficient $c_{\lambda}>0$ to each $\lambda \in \mathcal{L}^{p}$. These functions and coefficients are chosen in such a way that the operator $\vec{S}$ (formally) defined as

$$
\forall q=\sum_{\lambda \in \mathcal{L}^{p}} q_{\lambda} \psi_{\lambda}^{p}, \quad \vec{S} q:=\sum_{\lambda \in \mathcal{L}^{p}} c_{\lambda} q_{\lambda} \vec{\psi}_{\lambda}^{*}
$$

satisfies the following conditions:

i) $\vec{S}$ is bounded from $\mathcal{M}$ to $\vec{X}$; thus, there exists a constant $c_{*}>0$ such that

$$
\|\vec{S} q\|_{\vec{X}} \leq c_{*}\|q\|_{M}, \quad \forall q \in \mathcal{M}
$$

ii) there exists a constant $\beta_{*}>0$ such that

$$
-\langle\nabla q, \vec{S} q\rangle \geq \beta_{*}\|q\|_{M}^{2}, \quad \forall q \in \mathcal{M} ;
$$

iii) the orthogonality relation

$$
\left\langle\nabla q_{0}, \vec{S} q\right\rangle=0, \quad \forall q_{0} \in M_{0}, \forall q \in \mathcal{M}
$$

holds.

We shall see below how such conditions can be fulfilled.

Finally, let us select (by some adaptive procedure, that we will not detail here) a finite subset $\Lambda^{p} \subset \mathcal{L}^{p}$; let us set $\mathcal{M}_{\Lambda^{p}}=\operatorname{span}\left\{\psi_{\lambda}^{p}: \lambda \in \Lambda^{p}\right\}$ and $M_{\Lambda^{p}}=M_{0} \oplus \mathcal{M}_{\Lambda^{p}}$. Furthermore, let us select a finite-dimensional subspace $\vec{X}_{\Lambda^{v}} \subset \vec{X}$, containing the subspace $\vec{X}_{0}$ defined in (2.6) . Note that $\vec{X}_{\Lambda^{v}}$ need not contain any of the velocities $\vec{\psi}_{\lambda}^{*}$ which enter into the definition of the stabilizing operator $\vec{S}$.

We consider the following consistently stabilized Galerkin discretization of problem (2.1)-(2.3): Find $\vec{u} \in \vec{X}_{\Lambda^{v}}$ and $p \in M_{\Lambda^{p}}$ such that

$$
\begin{aligned}
(\nabla \vec{u}, \nabla \vec{v})-(\nabla \cdot \vec{v}, p) & =\langle\vec{f}, \vec{v}\rangle, \quad \forall \vec{v} \in \vec{X}_{\Lambda^{v}}, \\
(\nabla \cdot \vec{u}, q)+\delta\left\langle\vec{e} s, \vec{S} q_{\mathcal{M}}\right\rangle & =0, \quad \forall q \in M_{\Lambda^{p}},
\end{aligned}
$$

where $\delta>0$ is a suitable stabilization parameter,

$$
r \vec{e} s=\vec{f}+\Delta \vec{u}-\nabla p \in \vec{X}^{\prime}
$$

is the residual of the momentum equation, and pressures are split as $q=q_{0}+q_{\mathcal{M}} \in$ $M_{0} \oplus \mathcal{M}$ according to (2.4).

In order to study this problem, let us introduce the bilinear form $B:(\vec{X} \times M)^{2} \rightarrow$ $\mathbb{R}$ defined as

$$
B[(\vec{w}, r),(\vec{v}, q)]=(\nabla \vec{w}, \nabla \vec{v})-(\nabla \cdot \vec{v}, r)+(\nabla \cdot \vec{w}, q)+\delta\left\langle\Delta \vec{w}-\nabla r, \vec{S} q_{\mathcal{M}}\right\rangle
$$

as well as the linear form $F: \vec{X} \times M \rightarrow \mathbb{R}$ defined as

$$
F(\vec{v}, q)=\langle\vec{f}, \vec{v}\rangle-\delta\left\langle\vec{f}, \vec{S} q_{\mathcal{M}}\right\rangle .
$$


Then, problem (2.12)-(2.13) can be rewritten: Find $(\vec{u}, p) \in \vec{X}_{\Lambda^{v}} \times M_{\Lambda^{p}}$ such that

$$
B[(\vec{u}, p),(\vec{v}, q)]=F(\vec{v}, q), \quad \forall(\vec{v}, q) \in \vec{X}_{\Lambda^{v}} \times M_{\Lambda^{p}} .
$$

Note that the exact problem (2.1)-(2.3) can be equivalently written: Find $(\vec{U}, P) \in$ $\vec{X} \times M$ such that

$$
B[(\vec{U}, P),(\vec{v}, q)]=F(\vec{v}, q), \quad \forall(\vec{v}, q) \in \vec{X} \times M .
$$

Thanks to condition (2.9), both forms $B$ and $F$ are continuous on their spaces of definitions. The following result will guarantee existence and uniqueness of the solution of the above variational problems.

Proposition 2.1. Let $\delta$ satisfy the inequality

$$
\delta \leq \min \left(1, \frac{2 \beta_{*}}{2 c_{*}^{2}+\sigma_{0} \beta_{*}^{2}}\right),
$$

where $\sigma_{0}=0$ if $M_{0}=\emptyset, \sigma_{0}=1$ if $M_{0} \neq \emptyset$. Then there exists a constant $\beta>0$ (independent of $\delta$ ) such that

$$
\inf _{(\vec{w}, r) \in \overrightarrow{\mathrm{X}} \times \mathrm{M}} \sup _{(\vec{v}, q) \in \overrightarrow{\mathrm{X}} \times \mathrm{M}} \frac{B[(\vec{w}, r),(\vec{v}, q)]}{\|(\vec{w}, r)\|_{\vec{X} \times M}\|(\vec{v}, q)\|_{\vec{X} \times M}} \geq \beta,
$$

where $\overrightarrow{\mathrm{X}} \times \mathrm{M}$ equals $\vec{X} \times M$ or $\vec{X}_{\Lambda^{v}} \times M_{\Lambda^{p}}$, and the norm in $\vec{X} \times M$ is scaled as $\|(\vec{v}, q)\|_{\vec{X} \times M}^{2}=\|\vec{v}\|_{\vec{X}}^{2}+\delta\|q\|_{M}^{2}$.

Proof. Taking $(\vec{v}, q)=(\vec{w}, r) \in \overrightarrow{\mathrm{X}} \times \mathrm{M}$ and using (2.10) and (2.11), one gets

$$
\begin{aligned}
B[(\vec{w}, r),(\vec{w}, r)] & =(\nabla \vec{w}, \nabla \vec{w})-\delta\left\langle\nabla r_{\mathcal{M}}, \vec{S} r_{\mathcal{M}}\right\rangle+\delta\left\langle\Delta \vec{w}, \vec{S} r_{\mathcal{M}}\right\rangle \\
& \geq\|\vec{w}\|_{\vec{X}}^{2}+\delta \beta_{*}\left\|r_{\mathcal{M}}\right\|_{M}^{2}+\delta\left\langle\Delta \vec{w}, \vec{S} r_{\mathcal{M}}\right\rangle .
\end{aligned}
$$

On the other hand, by (2.9) and the Hölder inequality $|a b| \leq \frac{1}{2 \eta} a^{2}+\frac{\eta}{2} b^{2}$ with an appropriate choice of $\eta>0$, we have

$$
\begin{aligned}
\left|\left\langle\Delta \vec{w}, \vec{S} r_{\mathcal{M}}\right\rangle\right| & \leq\|\Delta \vec{w}\|_{\vec{X}},\left\|\vec{S} r_{\mathcal{M}}\right\|_{\vec{X}} \leq\|\vec{w}\|_{\vec{X}}\left\|\vec{S} r_{\mathcal{M}}\right\|_{\vec{X}} \\
& \leq c_{*}\|\vec{w}\|_{\vec{X}}\left\|r_{\mathcal{M}}\right\|_{M} \leq \frac{c_{*}^{2}}{2 \beta_{*}}\|\vec{w}\|_{\vec{X}}^{2}+\frac{\beta_{*}}{2}\left\|r_{\mathcal{M}}\right\|_{M}^{2} .
\end{aligned}
$$

Therefore, we obtain

$$
B[(\vec{w}, r),(\vec{w}, r)] \geq\left(1-\delta \frac{c_{*}^{2}}{2 \beta_{*}}\right)\|\vec{w}\|_{\vec{X}}^{2}+\delta \frac{\beta_{*}}{2}\left\|r_{\mathcal{M}}\right\|_{M}^{2} .
$$

This gives the desired result if $M_{0}=\emptyset$. From now on, let us assume that $M_{0} \neq \emptyset$. Recalling (2.6), let $\vec{v}_{0} \in \vec{X}_{0} \subset \overrightarrow{\mathrm{X}}$ be such that $\left(\nabla \cdot \vec{v}_{0}, r_{0}\right) \geq \beta_{0}\left\|\vec{v}_{0}\right\|_{\vec{X}}\left\|r_{0}\right\|_{M}$, with $\left\|\vec{v}_{0}\right\|_{\vec{X}}=\gamma\left\|r_{0}\right\|_{M}, \gamma>0$ to be defined. Then,

$$
\begin{aligned}
B\left[(\vec{w}, r),\left(-\vec{v}_{0}, 0\right)\right] & =-\left(\nabla \vec{w}, \nabla \vec{v}_{0}\right)+\left(\nabla \cdot \vec{v}_{0}, r_{\mathcal{M}}\right)+\left(\nabla \cdot \vec{v}_{0}, r_{0}\right) \\
& \geq-\gamma\|\vec{w}\|_{\vec{X}}\left\|r_{0}\right\|_{M}-\gamma\left\|r_{\mathcal{M}}\right\|_{M}\left\|r_{0}\right\|_{M}+\gamma \beta_{0}\left\|r_{0}\right\|_{M}^{2} .
\end{aligned}
$$

Using again appropriate Hölder inequalities for the first and second terms, we get

$$
B\left[(\vec{w}, r),\left(-\vec{v}_{0}, 0\right)\right] \geq-\frac{\gamma}{\beta_{0}}\|\vec{w}\|_{\vec{X}}^{2}-\frac{\gamma}{\beta_{0}}\left\|r_{\mathcal{M}}\right\|_{M}^{2}+\gamma \frac{\beta_{0}}{2}\left\|r_{0}\right\|_{M}^{2}
$$


Summing up, we obtain

$B\left[(\vec{w}, r),\left(\vec{w}-\vec{v}_{0}, r\right)\right] \geq\left(1-\delta \frac{c_{*}^{2}}{2 \beta_{*}}-\frac{\gamma}{\beta_{0}}\right)\|\vec{w}\|_{\vec{X}}^{2}+\left(\delta \frac{\beta_{*}}{2}-\frac{\gamma}{\beta_{0}}\right)\left\|r_{\mathcal{M}}\right\|_{M}^{2}+\gamma \frac{\beta_{0}}{2}\left\|r_{0}\right\|_{M}^{2}$.

Now, we choose $\gamma=\delta \frac{\beta_{*} \beta_{0}}{4}$. The conclusion follows, taking into account (2.4).

Proposition 2.1 allows us to establish classical abstract a priori and a posteriori error estimates between the solutions of problems (2.1)-(2.3) and (2.12)-(2.13). Since proofs are by now standard, we only give the results, in which the dependence upon the stabilization parameter $\delta$ has been made explicit.

Proposition 2.2. Under the conditions (2.5) -(2.6) on the pressure spaces, (2.9) (2.11) on the stabilizing operator $\vec{S}$ and (2.16) on the stabilization parameter $\delta$, the following a priori estimate holds:

$$
\begin{aligned}
\|\vec{U}-\vec{u}\|_{\vec{X}}+\delta^{1 / 2}\|P-p\|_{M} \lesssim & \inf _{\vec{v} \in \vec{X}_{\Lambda^{v}}}\left(\|\vec{U}-\vec{v}\|_{\vec{X}}+\delta^{-1 / 2}\|\nabla \vec{U}-\nabla \vec{v}\|_{M}\right) \\
& +\inf _{q \in M_{\Lambda^{p}}}\|P-q\|_{M} \cdot \square
\end{aligned}
$$

Proposition 2.3. Under the above conditions, the following a posteriori estimate holds:

$$
\begin{array}{ll}
\|\vec{U}-\vec{u}\|_{\vec{X}} & +\delta^{1 / 2}\|P-p\|_{M} \leq \sup _{\vec{V} \in \vec{X}} \inf _{\vec{v} \in \vec{X}_{\Lambda^{v}}} \frac{|\langle r \vec{e} s, \vec{V}-\vec{v}\rangle|}{\|\vec{V}\|_{\vec{X}}} \\
& +\sup _{Q \in M} \inf _{q \in M_{\Lambda^{p}}} \frac{\delta^{-1 / 2}|(\nabla \cdot \vec{u}, Q-q)|+\delta^{1 / 2}|\langle r \vec{e} s, \vec{S}(Q-q)\rangle|}{\|Q\|_{M}} .
\end{array}
$$

We end this section by indicating a natural way of choosing the auxiliary functions $\vec{\psi}_{\lambda}^{*}, \lambda \in \mathcal{L}^{p}$, in such a way that the crucial condition (2.10) is easily fulfilled.

Proposition 2.4. For any $\lambda \in \mathcal{L}^{p}$, let $\vec{\psi}_{\lambda}^{*} \in \vec{X}$ be such that

$$
\nabla \cdot \vec{\psi}_{\lambda}^{*}=\tilde{\psi}_{\lambda}^{p} \quad \text { in } \Omega
$$

Furthermore, set

$$
c_{\lambda}=1
$$

in the definition (2.8) of the operator $\vec{S}$. Then, conditions (2.10) and (2.11) are satisfied.

Proof. For any $q=\sum_{\lambda \in \mathcal{L}^{p}} q_{\lambda} \psi_{\lambda}^{p} \in \mathcal{M}$, we have

$$
-\langle\nabla q, \vec{S} q\rangle=(q, \nabla \cdot \vec{S} q)=\sum_{\lambda \in \mathcal{L}^{p}} q_{\lambda}\left(q, \nabla \cdot \vec{\psi}_{\lambda}^{*}\right)=\sum_{\lambda \in \mathcal{L}^{p}} q_{\lambda}^{2},
$$

and (2.10) follows from (2.5). On the other hand, 2.11) is a consequence of the inclusion $\tilde{\Psi}^{p} \subset M_{0}^{\perp}$.

Remark 2.5. The choice (2.21) for the coefficients of $\vec{S}$ is the simplest one from the theoretical point of view. A control of the $L^{2}(\Omega)$-norm of the pressure is assured as well if we relax the condition into $c_{\lambda} \sim 1, \forall \lambda \in \mathcal{L}^{p}$. This enhances flexibility and efficiency in our stabilization scheme, allowing for a local (in scale and position) tuning of the coefficients. 


\section{The CONSTRUCTION OF THE STABILIZING WAVELETS}

In this section, we shall construct a Riesz basis $\Psi^{p}$ for the pressure space, as well as its companion biorthogonal basis $\tilde{\Psi}^{p}$, for which it is easy to define auxiliary velocities fulfilling condition (2.20). At first, we consider the reference domain $\hat{\Omega}=] 0,1\left[{ }^{d}\right.$, where simple tensor product arguments can be used, starting from biorthogonal bases on the interval $(0,1)$ suitably modified at the endpoints. Next, we show how our construction can be transported on a domain $\Omega$, which is the smooth image of the reference domain. Finally, we deal with more general domains, using domain decomposition ideas.

3.1. Biorthogonal wavelets on the interval $] 0,1[$. As usual this construction is obtained by restriction to ]0,1[ and adaptation at the edges 0 and 1 of biorthogonal wavelets on the line. The present construction only differs from existing ones (see CDV], AJP for the basic ideas and also [DKU2], [Ma1, GT] for various specific topics) in our choice of $H_{0}^{1}(0,1)$ boundary conditions for the dual wavelets, while the primal wavelets (defining the pressure) have optimal order of approximation. This specific choice is needed in order to build the stabilizing wavelets $\vec{\psi}_{\lambda}^{*, \hat{\Omega}}$ by tensor products.

The construction on the line (see [CDF] ) starts from a pair of compactly supported scaling functions $(\phi, \tilde{\phi})$ of supports $\left[-m_{0}, m_{1}\right]$ and $\left[-\tilde{m}_{0}, \tilde{m}_{1}\right]$ (with integer edges) satisfying the two scale relations

$$
\begin{aligned}
& \phi=\sum_{m=-m_{0}}^{m_{1}} \sqrt{2} h_{m} \phi(2 .-m), \\
& \tilde{\phi}=\sum_{m=-\tilde{m}_{0}}^{\tilde{m}_{1}} \sqrt{2} \tilde{h}_{m} \tilde{\phi}(2 .-m)
\end{aligned}
$$

for finite masks $h$ and $\tilde{h}$, and the biorthogonality relations

$$
\langle\phi, \phi(x-k)\rangle=\delta_{k} \text { for all } k \in \mathbb{Z} .
$$

The primal and dual multiresolution analysis (MRA) spaces

$$
V_{j}(\mathbb{R}) \subset V_{j+1}(\mathbb{R}), \widetilde{V}_{j}(\mathbb{R}) \subset \widetilde{V}_{j+1}(\mathbb{R})
$$

are spanned by the biorthogonal compactly supported bases

$$
\begin{aligned}
& \Phi_{j}=\left\{\phi_{j, k}:=2^{\frac{j}{2}} \phi\left(2^{j} .-k\right), k \in \mathbb{Z}\right\}, \\
& \tilde{\Phi}_{j}=\left\{\tilde{\phi}_{j, k}:=2^{\frac{j}{2}} \tilde{\phi}\left(2^{j} .-k\right), k \in \mathbb{Z}\right\} .
\end{aligned}
$$

It is shown in $[\mathrm{CDF}]$ that the primal and dual wavelet spaces

$$
W_{j}(\mathbb{R}):=V_{j+1}(\mathbb{R}) \cap \widetilde{V}_{j}(\mathbb{R})^{\perp}, \widetilde{W}_{j}(\mathbb{R}):=\widetilde{V}_{j+1}(\mathbb{R}) \cap \widetilde{V}_{j}(\mathbb{R})^{\perp}
$$

are spanned by the biorthogonal compactly supported wavelet bases

$$
\Psi_{j}=\left\{2^{\frac{j}{2}} \psi\left(2^{j} \cdot-k\right), k \in \mathbb{Z}\right\}, \tilde{\Psi}_{j}=\left\{2^{\frac{j}{2}} \tilde{\psi}\left(2^{j} \cdot-k\right), k \in \mathbb{Z}\right\},
$$

where $\psi:=\sum \sqrt{2} g_{m} \phi(2 .-m)$ and $\tilde{\psi}:=\sum \sqrt{2} \tilde{g}_{m} \phi(2 .-m)$ are the mother wavelets obtained from the wavelet masks $g_{m}=(-1)^{m} \tilde{h}_{1-m}$ and $\tilde{g}_{m}=(-1)^{m} h_{1-m}$. The 
multiscale biorthogonal basis are defined (at least formally) by

$$
\Psi:=\Phi_{0} \cup \bigcup_{j \in \mathbb{N}} \Psi_{j} \text { and } \tilde{\Psi}:=\tilde{\Phi}_{0} \cup \bigcup_{j \in \mathbb{N}} \tilde{\Psi}_{j} ;
$$

it is convenient to denote them by $\Psi:=\left\{\psi_{\lambda}, \lambda \in \mathcal{L}\right\}, \tilde{\Psi}:=\left\{\tilde{\psi}_{\lambda}, \lambda \in \mathcal{L}\right\}$, where $\lambda$ stands for $(j, k)$ with $|\lambda|:=j$.

In $\mathrm{CDF}$ such compactly biorthogonal generators $(\phi, \tilde{\phi})$ are built with arbitrary smoothness. We denote by $\tau$ and $\tilde{\tau}$ the supremum of their smoothness measured in $H^{s}$. In addition, such generators can be built with arbitrary order of approximations $n$ and $\tilde{n}$ in the sense that the integer translates of $\phi$ and $\tilde{\phi}$ span the polynomials of $\mathbb{P}_{n-1}$ and $\mathbb{P}_{\tilde{n}-1}$.

Then it is shown that $\Psi$ (and symmetrically $\tilde{\Psi}$ ) is stable in $H^{s}(\mathbb{R}$ ) for the range $-\min (\tilde{\tau}, \tilde{n})<s<\min (\tau, n)$ in the sense that $\|f\|_{H^{s}(\mathbb{R})} \sim\left(\sum_{\lambda \in \mathcal{L}} 2^{2|\lambda| s}\left|\left(f, \tilde{\psi}_{\lambda}\right)\right|^{2}\right)^{1 / 2}$ for all $f \in H^{s}(\mathbb{R})$.

Hypothesis 3.1. We shall assume in the following that the pressure generators are chosen so that $n \geq 2, \tilde{n} \geq 2$ and $\tau>0, \tilde{\tau}>1$.

Starting from a pair of biorthogonal generators on the line $(\phi, \tilde{\phi})$, all the constructions of new MRA spaces $V_{j}$ and $\widetilde{V}_{j}$ on the interval share the basic ideas introduced in $\mathrm{CDV}$ and $\mathrm{AJP}$ to retain:

(i) the "interior" scaling functions on the line whose supports are in $\left[\mathrm{d}_{0} 2^{-j}, 1-\right.$ $\left.\mathrm{d}_{1} 2^{-j}\right]$ for $V_{j}$ and $\left[\tilde{\mathrm{d}}_{0} 2^{-j}, 1-\tilde{\mathrm{d}}_{1} 2^{-j}\right]$ for $\tilde{V}_{j}$, (where $\mathrm{d}=\left(\mathrm{d}_{0}, \mathrm{~d}_{1}\right), \tilde{\mathrm{d}}=\left(\tilde{\mathrm{d}}_{0}, \tilde{\mathrm{d}}_{1}\right)$ are pairs of nonnegative integer parameters);

(ii) at the edges 0 and 1 , only the $n$ (for $V_{j}$ ) and $\tilde{n}$ (for $\widetilde{V}_{j}$ ) truncated linear combinations of scaling functions that correspond to the reproduction on $] 0,1[$ of the monomials of degrees $\alpha=0, \ldots, n-1$ (for $V_{j}$ ) and $\alpha=0, \ldots, \tilde{n}-1$ (for $\widetilde{V}_{j}$ ).

Then, the optimal orders of approximation $n$ and $\tilde{n}$ and the nestedness are preserved.

This strategy enables us, in addition, to take into account homogeneous boundary conditions at the edges 0 or 1 for the primal or dual MRA. It suffices to retain in the previous definition only the monomials satisfying the same boundary conditions at the edges $\varepsilon=0,1$. For our purposes, the dual MRA will satisfy homogeneous Dirichlet boundary conditions at both edges while the primal MRA does not satisfy any boundary condition.

Recalling that $\operatorname{Supp} \phi=\left[-m_{0}, m_{1}\right]$, the primal MRA $V_{j}$ is defined as follows, with $\mathrm{CL}=\left(\mathrm{CL}_{0}, \mathrm{CL}_{1}\right)=(-1,-1)$ :

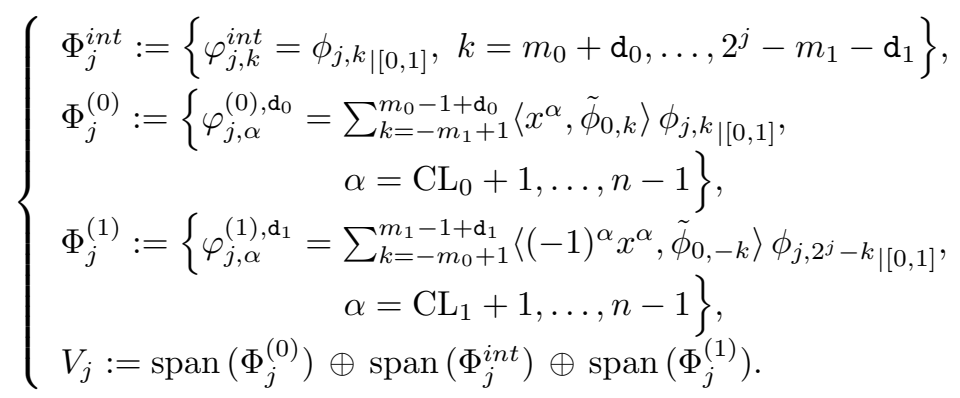


The dual MRA $\widetilde{V}_{j}$ is defined similarly with $\tilde{\mathrm{d}}=\left(\tilde{\mathrm{d}}_{0}, \tilde{\mathrm{d}}_{1}\right)$ and $\widetilde{\mathrm{CL}}=(0,0)$. These definitions of $V_{j}$ and $\widetilde{V}_{j}$ hold whenever $j \geq j_{0}$ for a coarse level $j_{0}$ such that there is no overlapping of the subscripts in the definitions of $\Phi_{j}^{i n t}$ and $\tilde{\Phi}_{j}^{i n t}$.

In order to obtain biorthogonal MRA spaces, the parameters $d$ and $\tilde{d}$ are chosen to match the dimensions of $V_{j}$ and $\widetilde{V}_{j}$ separately at both edges, i.e., for $\varepsilon=0,1$

$$
m_{\varepsilon}+\mathrm{d}_{\varepsilon}-n+\mathrm{CL}_{\varepsilon}=\tilde{m}_{\varepsilon}+\tilde{\mathrm{d}}_{\varepsilon}-\tilde{n}+\widetilde{\mathrm{CL}}_{\varepsilon} .
$$

Then, the MRA spaces admit local biorthogonal Riesz bases $\Phi_{j}$ and $\tilde{\Phi}_{j}$ if and only if two (one at each edge) fixed (independent of $j$ ) matrices are nonsingular, which will always be assumed in the following. This property is shown in DKU2 for spline biorthogonal wavelets.

The biorthogonal bases $\Phi_{j}$ and $\tilde{\Phi}_{j}$ will include a fixed number of modified scaling functions at the edges while all the other basis functions are scaling functions on the real line $\phi_{j, k}$ restricted to the interval.

The construction of local biorthogonal Riesz bases $\left(\Psi_{j}, \tilde{\Psi}_{j}\right)$ of the wavelet spaces $W_{j}:=V_{j+1} \cap \widetilde{V}_{j}^{\perp}, \widetilde{W}_{j}:=\widetilde{V}_{j+1} \cap V_{j}^{\perp}$ leads to a fixed number of locally supported modified wavelets at the edges while all the other wavelets are restrictions of wavelets on the real line (3.2). In addition, the modified wavelets at the edges are still dyadic dilate functions. For example, the primal wavelet basis writes

$$
\begin{aligned}
\Psi_{j} & :=\left\{\psi_{j, \alpha}: \alpha=0, \ldots, \nu_{0}\right\} \\
& \cup\left\{\psi_{j, k}:=2^{j / 2} \psi\left(2^{j} .-k\right): k=\nu_{0}+1, \ldots, 2^{j}-\nu_{1}-2\right\} \\
& \cup\left\{\psi_{j, 2^{j}-\beta-1}: \beta=0, \ldots, \nu_{1}\right\}
\end{aligned}
$$

with $\psi_{j+1, \alpha}=\sqrt{2} \psi_{j, \alpha}(2$.$) and \psi_{j+1,2^{j+1}-\beta-1}(1-)=.\sqrt{2} \psi_{j, 2^{j}-\beta-1}(1-2$.$) .$

In order to prove the boundedness of the stabilizing operator defined in the next subsection, we need to extend Meyer's "vaguelette lemma" stated on the line to the interval ]0,1[. Let us first recall the lemma on the line (see, e.g., $\mathrm{Me}$ ).

Lemma 3.2. Let $\eta$ be a compactly supported function such that $\eta \in H^{\sigma}(\mathbb{R})$ for some $\sigma>0$ and $\int_{\mathbb{R}} \eta=0$. Then for all $f \in L^{2}(\mathbb{R})$

$$
\sum_{j, k \in \mathbb{Z}}\left|\left(f, 2^{j / 2} \eta\left(2^{j} .-k\right)\right)\right|^{2} \lesssim\|f\|_{L^{2}(\mathbb{R})}^{2},
$$

or equivalently

$$
\left.\| \sum_{j, k \in \mathbb{Z}} d_{j, k} 2^{j / 2} \eta\left(2^{j} .-k\right)\right) \|_{L^{2}(\mathbb{R})}^{2} \lesssim \sum_{j, k \in \mathbb{Z}}\left|d_{j, k}\right|^{2} .
$$

Lemma 3.3. Let $\eta$ be a compactly supported function such that $\eta \in H^{\sigma}(\mathbb{R})$ for some $\sigma>0$ and $\int_{\mathbb{R}} \eta=0$. Moreover, let $\eta_{\alpha}^{(0)}, \alpha=0, \ldots, \nu_{0}$, be compactly supported functions of $H^{\sigma}(] 0, \infty[)$; similarly, let $\eta_{\beta}^{(1)}, \beta=0, \ldots, \nu_{1}$, be compactly supported functions of $H^{\sigma}(]-\infty, 1[)$. For $j \geq j_{0}$, let us set $\mathcal{K}_{j}:=\left\{0, \ldots, 2^{j}-1\right\}$ and, for any $k \in \mathcal{K}_{j}$,

$$
\eta_{j, k}:=2^{j / 2} \begin{cases}\eta_{k}^{(0)}\left(2^{j} .\right), & \text { if } k=0, \ldots, \nu_{0} \\ \eta\left(2^{j} \cdot-k\right), & \text { if } k=\nu_{0}+1, \ldots, 2^{j}-\nu_{1}-2, \\ \eta_{2^{j}-k-1}^{(1)}\left(1-2^{j}(1-.)\right), & \text { if } k=2^{j}-\nu_{1}-1 .\end{cases}
$$


Then for all $f \in L^{2}(] 0,1[)$

$$
\sum_{j \geq j_{0}, k \in \mathcal{K}_{j}}\left|\left(f, \eta_{j, k}\right)\right|^{2} \lesssim\|f\|_{L^{2}(] 0,1[)}^{2}
$$

or equivalently

$$
\left\|\sum_{j \geq j_{0}, k \in \mathcal{K}_{j}} d_{j, k} \eta_{j, k}\right\|_{L^{2}(] 0,1[)}^{2} \lesssim \sum_{j \geq j_{0}, k \in \mathcal{K}_{j}}\left|d_{j, k}\right|^{2} .
$$

Proof. Let $\tilde{f}$ the extension by 0 of $f$ to $\mathbb{R}$. Applying Lemma 3.2 one has

$$
\begin{aligned}
\sum_{j \geq j_{0}, k \in\left\{\nu_{0}+1, \ldots, 2^{j}-\nu_{1}-2\right\}}\left|\left(f, \eta_{j, k}\right)\right|^{2} & \leq \sum_{j, k \in \mathbb{Z}}\left|\left(\tilde{f}, 2^{j / 2} \eta\left(2^{j} \cdot-k\right)\right)\right|^{2} \\
& \lesssim\|\tilde{f}\|_{L^{2}(\mathbb{R})}^{2}=\|f\|_{L^{2}(] 0,1[)}^{2}
\end{aligned}
$$

On the other hand, one can always extend the functions $\eta_{\alpha}^{(0)}, \eta_{\beta}^{(1)}$ on the line so that these extensions are compactly supported functions of $H^{\sigma}(\mathbb{R})$ with vanishing mean values. Applying again Lemma 3.2 to these extensions yields (3.5). Finally, by transposition of the operator $f \rightarrow\left(f, \eta_{j, k}\right)_{j \geq j_{0}, k \in \mathcal{K}_{j}}$, property (3.6) is equivalent to (3.5).

For example, applying Lemma 3.3 to the primal and dual wavelet bases $\Psi:=$ $\Phi_{j_{0}} \cup \bigcup_{j \geq j_{0}} \Psi_{j}$ and $\tilde{\Psi}:=\tilde{\Phi}_{j_{0}} \cup \bigcup_{j \geq j_{0}} \tilde{\Psi}_{j}$, we deduce that they are biorthogonal Riesz bases. Using the combination of inverse and direct estimates in $V_{j}$ and interpolation theory will yield more precisely the stability of $\Psi$ in $H_{\mathrm{CL}}^{s}(] 0,1[)$ for the range $0 \leq s<\min (\tau, n)$ and symmetrically for $\tilde{\Psi}$ (see, e.g., [Co, [Da1]).

3.2. Pressure and stabilizing wavelets on the reference domain. Let us consider the biorthogonal MRA in $L^{2}(\hat{\Omega})$

$$
V_{j}(\hat{\Omega})=\bigotimes_{m=1}^{d} V_{j}, \widetilde{V}_{j}(\hat{\Omega})=\bigotimes_{m=1}^{d} \widetilde{V}_{j} .
$$

The corresponding wavelet spaces $W_{j}(\hat{\Omega})$ (and similarly $\widetilde{W}_{j}(\hat{\Omega})$ ) are spanned by the tensor product wavelet bases

$$
\Psi_{j}^{p, \hat{\Omega}}:=\bigcup_{\varepsilon \in \mathcal{E}} \bigotimes_{m=1}^{d} \Theta_{j}^{\varepsilon_{m}}, \quad j \geq j_{0},
$$

where $\mathcal{E}:=\{0,1\}^{d} /\{(0, \ldots, 0)\}$ and $\Theta_{j}^{\varepsilon_{m}}=\Phi_{j}$ if $\varepsilon_{m}=0, \Theta_{j}^{\varepsilon_{m}}=\Psi_{j}$ if $\varepsilon_{m}=1$.

We decompose $V_{j_{0}}(\hat{\Omega})$ as

$$
V_{j_{0}}(\hat{\Omega})=\operatorname{span}\{1\} \oplus \hat{M}_{j_{0}}
$$

and correspondingly

$$
\widetilde{V}_{j_{0}}(\hat{\Omega})=\operatorname{span}\left\{\tilde{\phi}_{0}\right\} \oplus \widetilde{\hat{M}}_{j_{0}}
$$

with $\widetilde{\hat{M}}_{j_{0}} \perp 1$ and $\hat{M}_{j_{0}} \perp \tilde{\phi}_{0}$. Then we choose

$$
\hat{\mathcal{M}}:=\hat{M}_{j_{0}} \oplus \bigoplus_{j \geq j_{0}} W_{j}(\hat{\Omega})
$$


so that the primal and dual pressure wavelet bases read

$$
\Psi^{p, \hat{\Omega}}:=\Phi_{j_{0}}^{p, \hat{\Omega}} \cup \bigcup_{j \geq j_{0}} \Psi_{j}^{p, \hat{\Omega}}, \quad \tilde{\Psi}^{p, \hat{\Omega}}:=\tilde{\Phi}_{j_{0}}^{p, \hat{\Omega}} \cup \bigcup_{j \geq j_{0}} \tilde{\Psi}_{j}^{p, \hat{\Omega}},
$$

for a given choice of biorthogonal bases $\left(\Phi_{j_{0}}^{p, \hat{\Omega}}, \tilde{\Phi}_{j_{0}}^{p, \hat{\Omega}}\right)$ of $\left(\hat{M}_{j_{0}}, \widetilde{\hat{M}}_{j_{0}}\right)$. For the construction of the stabilizing wavelets at level $j_{0}$ it is convenient to choose a tensor product basis for $\tilde{\Phi}_{j_{0}}^{p, \hat{\Omega}}$. For example in dimension two, let us assume that $\tilde{\Phi}_{j}=\left\{\tilde{\varphi}_{j, k}, k=1, \ldots, K_{j}\right\}$ with $\int_{0}^{1} \tilde{\varphi}_{j, k}(s) d s=C 2^{-j / 2}$ for all $k$, then we set

$$
\begin{aligned}
\tilde{\Phi}_{j_{0}}^{p, \hat{\Omega}}:= & \left\{\left(\tilde{\varphi}_{j_{0}, k}-\tilde{\varphi}_{j_{0}, k-1}\right) \otimes \tilde{\varphi}_{j_{0}, k^{\prime}}\right\}_{k=2, \ldots, K_{j}, k^{\prime}=1, \ldots, K_{j}} \\
& \cup\left\{\tilde{\varphi}_{j_{0}, 1} \otimes\left(\tilde{\varphi}_{j_{0}, k^{\prime}}-\tilde{\varphi}_{j_{0}, k^{\prime}-1}\right)\right\}_{k^{\prime}=2, \ldots, K_{j}} .
\end{aligned}
$$

In the following it is convenient to use the notation $\Psi^{p, \hat{\Omega}}=\left\{\psi_{\lambda}^{p, \hat{\Omega}}: \lambda \in \hat{\mathcal{L}}^{p}\right\}$, $\tilde{\Psi}^{p, \hat{\Omega}}=\left\{\tilde{\psi}_{\lambda}^{p, \hat{\Omega}}: \lambda \in \hat{\mathcal{L}}^{p}\right\}$.

Stabilizing wavelets. Given any $\tilde{\psi}_{\lambda}^{p, \hat{\Omega}} \in \tilde{\Psi}^{p, \hat{\Omega}}$, one has for at least one index (chosen arbitrarily) $m(\lambda) \in\{1, \ldots, d\}$

$$
\tilde{\psi}_{\lambda}^{p, \hat{\Omega}}(\hat{x}):=\tilde{\theta}\left(\hat{x}_{m(\lambda)}\right) \otimes \bigotimes_{l \neq m(\lambda)} \tilde{\xi}_{l}\left(\hat{x}_{l}\right)
$$

with $\int_{0}^{1} \tilde{\theta}(s) d s=0, \tilde{\xi}_{l}(0)=\tilde{\xi}_{l}(1)=0$ and $\|\tilde{\theta}\|_{L^{2}(] 0,1[)} \sim\left\|\tilde{\xi}_{l}\right\|_{L^{2}(] 0,1[)} \sim 1$. Then, we define for all $\lambda \in \hat{\mathcal{L}}^{p}$

$$
\left\{\begin{array}{l}
\vec{\psi}_{\lambda}^{*, \hat{\Omega}}=\left(0, \ldots, \psi_{\lambda}^{*, \hat{\Omega}}, \ldots, 0\right), \quad \text { where } \\
\psi_{\lambda}^{*, \hat{\Omega}}(\hat{x})=\left(\int_{0}^{\hat{x}_{m(\lambda)}} \tilde{\theta}(s) d s\right) \otimes \bigotimes_{l \neq m(\lambda)} \tilde{\xi}_{l}\left(\hat{x}_{l}\right) .
\end{array}\right.
$$

Each $\vec{\psi}_{\lambda}^{*, \hat{\Omega}}$ belongs to $\left(H_{0}^{1}(\hat{\Omega})\right)^{d}$ and satisfies the condition

$$
\nabla \cdot \vec{\psi}_{\lambda}^{*, \hat{\Omega}}=\tilde{\psi}_{\lambda}^{p, \hat{\Omega}} \quad \text { in } \hat{\Omega} .
$$

Using these velocity wavelets, we introduce the stabilizing operator $\hat{S}$ defined as $\hat{S} \hat{q}=\sum_{\lambda \in \hat{\mathcal{L}}^{p}} \hat{q}_{\lambda} \vec{\psi}_{\lambda}^{*, \hat{\Omega}}$.

Proposition 3.4. $\hat{S}$ is bounded from $\hat{\mathcal{M}}$ to $\left(H_{0}^{1}(\hat{\Omega})\right)^{d}$.

Proof. By transposition (as for the vaguelette lemma), it is equivalent to prove that for all $v \in L^{2}(\hat{\Omega})$

$$
\sum_{i=1}^{d} \sum_{\lambda \in \hat{\mathcal{L}}^{p}}\left|\left(v, \partial_{x_{i}} \psi_{\lambda}^{*, \hat{\Omega}}\right)\right|^{2} \lesssim\|v\|_{L^{2}(\hat{\Omega})}^{2}
$$

For $j \geq j_{0}$, let $\Upsilon_{j}$ denote any one of the collections of $L^{2}(] 0,1[)$-functions $\tilde{\Psi}_{j}$ or $2^{-j} \frac{d}{d s} \tilde{\Psi}_{j}$ or $2^{j} \int_{0}^{s} \tilde{\Psi}_{j}$. Similarly let $\Xi_{j}$ denote any one of the collections of $L^{2}(] 0,1[)$ functions $\tilde{\Phi}_{j}$ or $2^{-j} \frac{d}{d s} \tilde{\Phi}_{j}$. From Lemma 3.3 and Hypothesis 3.1, the collections $\Upsilon_{j}$ satisfy for all $f \in L^{2}(] 0,1[)$

$$
\sum_{j \geq j_{0}}\left\|\left(f, \Upsilon_{j}\right)\right\|_{l^{2}}^{2} \lesssim\|f\|_{L^{2}(] 0,1[)}^{2}
$$


where $\left(f, \Upsilon_{j}\right)$ denote the vector of scalar products with the basis functions. On the other hand, from the local supports of the basis functions of $\Xi_{j}$ and Hypothesis 3.1 one has for all $f \in L^{2}(] 0,1[)$

$$
\left\|\left(f, \Xi_{j}\right)\right\|_{l^{2}}^{2} \lesssim\|f\|_{L^{2}(] 0,1[)}^{2} .
$$

For $|\lambda|=j$ fixed, the collection of wavelets $\left\{\partial_{x_{i}} \psi_{\lambda}^{*, \hat{\Omega}}\right\}$ splits into $\left(d^{2}-1\right)$ blocks, each of them being a tensor product of at least one of the $\Upsilon_{j}$ type and at most $d-1$ of the $\Xi_{j}$-type. Hence, we can easily conclude with a tensor product argument.

Remark 3.5. As pressure wavelet bases, we could have also considered tensor products of univariate wavelet bases. Let $(\Psi, \tilde{\Psi})$ be the univariate wavelet bases on the interval, then we set

$$
\Psi^{p, \hat{\Omega}}:=\left(\otimes^{d} \Psi / \otimes^{d} \Phi_{j_{0}}\right) \cup \Phi_{j_{0}}^{p, \hat{\Omega}}, \tilde{\Psi}^{p, \hat{\Omega}}:=\left(\otimes^{d} \tilde{\Psi} / \otimes^{d} \tilde{\Phi}_{j_{0}}\right) \cup \tilde{\Phi}_{j_{0}}^{p, \hat{\Omega}} .
$$

The stabilizing wavelets are defined as in (3.7) where in this case, for $\lambda \in \hat{\mathcal{L}}^{p}$, $\lambda:=\left(j_{l}, k_{l}, l=1, \ldots, d\right)$, the index $m(\lambda)$ is chosen so that it corresponds to the maximum scale $j_{l}, l=1, \ldots, d$. The boundedness of $\hat{S}$ is proven similarly by a slightly modified tensor product argument.

3.3. Extension by mapping. Let us assume that $\Omega$ is such that there exists a regular one-to-one parametrization $\kappa$ from $\overline{\hat{\Omega}}$ to $\bar{\Omega}$. We denote by $J$ the Jacobian matrix $\partial \kappa^{-1}$.

Let us set $M=\mathcal{M}:=\left\{q \circ \kappa^{-1}, q \in \hat{\mathcal{M}}\right\} \simeq L^{2}(\Omega) / \mathbb{R}$; consequently, let $M_{0}$ be empty. The push forward

$$
\Psi^{p}:=\left\{\psi_{\lambda}^{p, \hat{\Omega}} \circ \kappa^{-1}, \lambda \in \hat{\mathcal{L}}^{p}\right\}
$$

is a wavelet Riesz basis of $M$ and

$$
\tilde{\Psi}^{p}:=\left\{|J| \tilde{\psi}_{\lambda}^{p, \hat{\Omega}} \circ \kappa^{-1}, \lambda \in \hat{\mathcal{L}}^{p}\right\}
$$

is its dual Riesz basis with vanishing mean value. The following lemma is a key ingredient in the construction of the stabilizing wavelets.

Lemma 3.6. For any $\lambda \in \hat{\mathcal{L}}^{p}$, the Piola transform of $\vec{\psi}_{\lambda}^{*, \hat{\Omega}}$, i.e., the velocity defined as $\vec{\psi}_{\lambda}^{*}:=|J| J^{-1}\left(\vec{\psi}_{\lambda}^{*, \hat{\Omega}} \circ \kappa^{-1}\right) \in\left(H_{0}^{1}(\Omega)\right)^{d}$, satisfies

$$
\nabla \cdot \vec{\psi}_{\lambda}^{*}=\tilde{\psi}_{\lambda}^{p} \quad \text { in } \Omega
$$

Proof. By integration by part and a simple change of variable it is easily checked that for any $q \in L^{2}(\Omega)$

$$
\left(q, \nabla \cdot \vec{\psi}_{\lambda}^{*}\right)_{L^{2}(\Omega)}=-\left(\nabla(q \circ \kappa), \vec{\psi}_{\lambda}^{*, \hat{\Omega}}\right)_{\left(L^{2}(\hat{\Omega})\right)^{d}}=\left(q \circ \kappa, \tilde{\psi}_{\lambda}^{p, \hat{\Omega}}\right)_{L^{2}(\hat{\Omega})}=\left(q, \tilde{\psi}_{\lambda}^{p}\right)_{L^{2}(\Omega)} .
$$

We can now state the following result.

Proposition 3.7. The stabilizing operator $S$ defined by (2.8) and (2.21) satisfies properties (2.9), (2.10) and (2.11).

Proof. The bound (2.9) can be easily obtained from Proposition 3.4 and the regularity of the parametrization. Property (2.10) derives from Lemma 3.6 and Proposition 2.4. Property (2.11) is trivial. 
3.4. Extension by domain decomposition. Finally, we consider the case in which $\Omega$ is decomposable (not necessarily in a conforming way) into the union of disjoint subdomains $\Omega_{i}, i=1, \ldots, N$ with $\bar{\Omega}=\bigcup_{i=1}^{N} \bar{\Omega}_{i}$. Each $\Omega_{i}$ is the image of the reference domain under a smooth mapping $\kappa_{i}$, as in the previous subsection.

For $i=1, \ldots, N$, let $\mathcal{M}_{i}$ be the space of pressures in $\Omega_{i}$, obtained from $\hat{\mathcal{M}}$ by the mapping procedure described above. Let $\Psi_{i}^{p}$ and $\tilde{\Psi}_{i}^{p}$ be the corresponding biorthogonal Riesz bases. Then, we set

$$
\mathcal{M}:=\bigoplus_{i=1}^{N} \mathcal{M}_{i} .
$$

The wavelet Riesz basis $\Psi^{p}$ of $\mathcal{M}$ is defined by taking the union of the local Riesz bases extended by zero outside their subdomain of construction. The dual Riesz basis $\tilde{\Psi}^{p}$ is defined similarly. Note that this choice leads to discontinuous pressure wavelets at the interfaces of the decomposition, whereas the dual basis functions are in $H_{0}^{1}(\Omega)$. For simplicity we have taken the same reference wavelet basis $\Psi^{p, \hat{\Omega}}$ on $\hat{\mathcal{M}}$ for each subdomain $\Omega_{i}, i=1, \ldots, N$. The analysis would obviously extend to different choices of the reference wavelet bases for each subdomain.

Let $M_{0} \subset L^{2}(\Omega) / \mathbb{R}$ be the subspace of the pressures which are piecewise constant on each subdomain. Then we set

$$
M:=M_{0} \oplus \mathcal{M} \simeq L^{2}(\Omega) / \mathbb{R} .
$$

Note that the dual pressure basis $\tilde{\Psi}^{p}$ is orthogonal to $M_{0}$.

The stabilizing velocities are defined as follows. For each subdomain $\Omega_{i}$, we consider the velocities defined via the Piola transform as in Lemma 3.6 and we extend them by zero outside the subdomain. It is immediate that the velocities so obtained are in $\vec{X}$, and Proposition 3.7 holds as well.

Thus, we are left with the inf-sup condition (2.6). We now give a fairly general condition on the discrete velocity space $\vec{X}_{\Lambda^{v}}$, which guarantees its validity. Taking into account the definition of $M_{0}$, we can easily adapt to the present situation an argument, based on Fortin's lemma, which is classical in the finite element theory (see, e.g., $[\mathrm{BF}]$ ). For the reader's convenience, we report the details.

We aim at defining a linear operator $\Pi_{\Lambda^{v}}: \vec{X} \rightarrow \vec{X}_{\Lambda^{v}}$ such that

$$
\left\|\Pi_{\Lambda^{v}} \vec{v}\right\|_{\vec{X}} \lesssim\|\vec{v}\|_{\vec{X}}, \quad \forall \vec{v} \in \vec{X},
$$

and

$$
\left(\nabla \cdot\left(\vec{v}-\Pi_{\Lambda^{v}} \vec{v}\right), q\right)_{L^{2}(\Omega)}=\sum_{i=1}^{N} q_{\mid \Omega_{i}} \int_{\Omega_{i}} \nabla \cdot\left(\vec{v}-\Pi_{\Lambda^{v}} \vec{v}\right) d x=0, \quad \forall q \in M_{0} .
$$

The latter identities hold if

$$
\int_{\partial \Omega_{i}} \Pi_{\Lambda^{v}} \vec{v} \cdot \vec{n} d \gamma=\int_{\partial \Omega_{i}} \vec{v} \cdot \vec{n} d \gamma, \quad \forall \vec{v} \in \vec{X}, \quad i=1, \ldots, N .
$$

In order to fulfill these conditions, let us set $\Gamma_{i j}:=\partial \Omega_{i} \cap \partial \Omega_{j}$ and let us define the index set

$$
\mathcal{I}:=\left\{(i, j): 1 \leq i<j \leq N \text { and }\left|\Gamma_{i, j}\right|>0\right\} .
$$

Let us make the following 
Hypothesis 3.8. For any $(i, j) \in \mathcal{I}$, there exists $\vec{v}^{(i, j)} \in \vec{X}_{\Lambda^{v}}$ such that

$$
\left\|\vec{v}^{(i, j)}\right\|_{\vec{X}} \lesssim 1, \quad \vec{v}^{(i, j)} \text { is supported in } \overline{\Omega_{i} \cup \Omega_{j}}, \quad \int_{\Gamma_{i j}} \vec{v}^{(i, j)} \cdot \vec{n}^{(i, j)} d \gamma=1,
$$

where $\vec{n}^{(i, j)}$ denotes the normal unit vector oriented from $\Omega_{i}$ to $\Omega_{j}$.

Let us define

$$
\Pi_{\Lambda^{v}} \vec{v}:=\sum_{(i, j) \in \mathcal{I}}\left(\int_{\Gamma_{i j}} \vec{v} \cdot \vec{n}^{(i, j)} d \gamma\right) \vec{v}^{(i, j)} .
$$

Then, it is immediate to check that (3.10) holds. On the other hand, setting $\mathcal{I}(i, j):=\{(l, m) \in \mathcal{I}:\{l, m\} \cap\{i, j\} \neq \emptyset\}$, we have

$$
\begin{aligned}
\left\|\Pi_{\Lambda^{v}} \vec{v}\right\|_{\vec{X}}^{2}= & \sum_{(i, j) \in \mathcal{I}} \sum_{(l, m) \in \mathcal{I}}\left(\int_{\Gamma_{i j}} \vec{v} \cdot \vec{n}^{(i, j)} d \gamma\right) \\
& \times\left(\int_{\Gamma_{l m}} \vec{v} \cdot \vec{n}^{(l, m)} d \gamma\right)\left(\vec{v}^{(i, j)}, \vec{v}^{(l, m)}\right)_{\vec{X}} \\
\lesssim & \sum_{(i, j) \in \mathcal{I}} \sum_{(l, m) \in \mathcal{I}(i, j)}\left|\int_{\Gamma_{i j}} \vec{v} \cdot \vec{n}^{(i, j)} d \gamma\right|\left|\int_{\Gamma_{l m}} \vec{v} \cdot \vec{n}^{(l, m)} d \gamma\right| \\
\lesssim & \sum_{(i, j) \in \mathcal{I}} \sum_{(l, m) \in \mathcal{I}(i, j)}\|\vec{v}\|_{\left(H^{1}\left(\Omega_{i}\right)\right)^{d}}\|\vec{v}\|_{\left(H^{1}\left(\Omega_{l}\right)\right)^{d} .}
\end{aligned}
$$

By observing that $\operatorname{card} \mathcal{I}(i, j) \lesssim 1$ independently of the number of subdomains, we easily get (3.9). The constant implied by the symbol $\lesssim$ is independent of $\Lambda^{v}$ and the number of subdomains.

We summarize our result in the following proposition.

Proposition 3.9. Let Hypothesis 3.8 be satisfied, and let us define

$$
\vec{X}_{0}:=\operatorname{span}\left\{\vec{v}^{(i, j)}:(i, j) \in \mathcal{I}\right\} .
$$

Then, the inf-sup condition (2.6) is fulfilled.

\section{Numerical Results}

We aim at solving the Stokes problem in the square $\Omega=\hat{\Omega}=] 0,1\left[^{2}\right.$. Although the main interest of our stabilization method concerns its use in an adaptive framework, hereafter for simplicity, we shall only consider uniform discretization spaces $\vec{X}_{\Lambda^{v}}:=$ $\vec{X}_{J_{v}}$ for the velocity and $M_{\Lambda^{p}}:=M_{J_{p}}$ for the pressure, where $J_{v}$ and $J_{p}$ are suitable level indices.

Precisely, the velocity discretization is $\vec{X}_{J_{v}}:=X_{J_{v}} \times X_{J_{v}}$, where $X_{J_{v}}$ is the $\mathbb{Q}_{1}$ finite element space (with $H_{0}^{1}$ boundary conditions) on the dyadic grid $\left\{\left(2^{-J_{v}} k_{x}\right.\right.$, $\left.\left.2^{-J_{v}} k_{y}\right), k_{x}, k_{y}=0, \ldots, 2^{J_{v}}\right\}$. Equivalently, $X_{J_{v}}$ is the tensor product MRA space obtained from the spline generator on the line $\phi^{2}:=\left(\chi_{[0,1]}\right)^{* 2}$. A wavelet decomposition is obtained on $X_{J_{v}}$ from the spline biorthogonal wavelet generator on the line $\psi^{2, \tilde{n}_{v}}$ which corresponds to the choice of a dual generator $\tilde{\phi}^{2, \tilde{n}_{v}}\left(\tilde{n}_{v}\right.$ being an appropriate index, see [CDF]).

As far as the pressure is concerned, let $\left(V_{j}, \widetilde{V}_{j}\right)$ be the biorthogonal MRA on the interval $[0,1]$ obtained, as described in Section[3, from the spline biorthogonal generators on the line $\left(\phi^{2}, \tilde{\phi}^{2, \tilde{n}_{p}}\right)$ (for a suitable $\tilde{n}_{p}$ ), with $\mathrm{d}_{0}=\mathrm{d}_{1}=2$ and $\tilde{\mathrm{d}}_{0}=$ 
$\tilde{\mathrm{d}}_{1}=0$. This choice of $(\mathrm{d}, \tilde{\mathrm{d}})$ is imposed by the $H_{0}^{1}$ boundary conditions on the dual MRA $\widetilde{V}_{j}$. It results that the dimension of the pressure MRA on the interval is $2^{j}-1$ rather than $2^{j}+1$. The pressure space $M_{J_{p}} \simeq V_{J_{p}} \otimes V_{J_{p}} / \mathbb{R}$ and its dual $\widetilde{M}_{J_{p}}:=\left\{\tilde{q} \in \widetilde{V}_{J_{p}} \otimes \widetilde{V}_{J_{p}}, \int_{\hat{\Omega}} \tilde{q}=0\right\}$ are obtained from these MRA on the interval as described in Section 3 , We shall use in the following experiment tensor product stabilizing wavelets $\vec{\psi}_{\lambda}^{*} \hat{\Omega}$ as given by Remark 3.5

About the level indices, let us observe that for $J_{v}=J_{p}:=J$, the nonstabilized approximation admits no spurious mode (due to the dimension $2^{J}-1$ of $V_{J}$ ), although the inf-sup constant behaves like $2^{-J}$. Hence, to illustrate the efficiency of the stabilization, we will rather be interested in the cases $J_{p}>J_{v}$ for which the nonstabilized approximation is not well posed.

The velocity and pressure test and trial functions are expressed in their scaling function bases. Only for the computation of the stabilizing term, we resort to wavelet decompositions of the pressure and the auxiliary velocity. The resulting asymptotic complexity of this term is $\mathcal{O}\left(N(\log (N))^{2}\right)$ where $N:=2^{2 \max \left(J_{v}, J_{p}\right)}$. Note that it could be further reduced to $N$ by using an appropriate wavelet compression (see, e.g., DPS]).

The wavelet bases scalar products involved in the stabilizing terms are computed within round-off error solving small eigenvalue-eigenvector problems (see [DM]). For that purpose we need a third auxiliary MRA obtained from the compactly supported spline biorthogonal generators on the line $\left(\phi^{1}, \tilde{\phi}^{1, \tilde{n}_{p}+1}\right)$ (see [CDF] for their definition). The corresponding wavelets $\left(\psi^{1, \tilde{n}_{p}+1}, \tilde{\psi}^{1, \tilde{n}_{p}+1}\right)$ satisfy the properties

$$
\tilde{\psi}^{1, \tilde{n}_{p}+1}(x)=\frac{1}{4} \int_{-\infty}^{x} \tilde{\psi}^{2, \tilde{n}_{p}}(t) d t, \quad \frac{d}{d x} \psi^{2, \tilde{n}_{p}+1}=-4 \psi^{1, \tilde{n}_{p}}
$$

(see [L] for details).

Let us denote by $\mathcal{A}, \mathcal{G}, \mathcal{D}$ and $\mathcal{S}$, respectively, the matrices in scaling function bases for the Galerkin Laplacian, gradient, divergence and stabilizing operator $\vec{S}$, respectively. The Galerkin matrix in scaling function bases of the operator $H:=$ $-\vec{S}^{*} \Delta$ from $\mathcal{M}$ to $\vec{X}^{\prime}$ is denoted by $\mathcal{H}$.

By construction, the matrix $\mathcal{C}:=\mathcal{D S}$ is equal to $\mathcal{T}^{T} \mathcal{T}$, where $\mathcal{T}$ is the transformation computing the components of the pressure in the wavelet basis from its components in the scaling function basis. Hence, the matrix-vector products $\mathcal{C} Q$ and $\mathcal{C}^{-1} \tilde{Q}$ are computed in $\mathcal{O}\left(2^{2 J_{p}}\right)$ operations by two fast wavelet transforms.

With these notations, the stabilized Stokes system amounts to finding vectors $U$ and $P$ such that

$$
\left\{\begin{array}{llc}
\mathcal{A} U+\mathcal{G} P & =F \\
\mathcal{D} U-\delta \mathcal{H} U+\delta \mathcal{C} P & = & -\delta \mathcal{S} F
\end{array}\right.
$$

In order to solve this system efficiently, we eliminate the pressure in the continuity equation and substitute the pressure in the momentum equation. Furthermore, in order to recover the symmetry we add the consistent term $-\mathcal{H}^{T} \mathcal{C}^{-1} \mathcal{D} U$ to the resulting equation. This yields a symmetric system for the velocity $U$, namely

$$
\left(\mathcal{A}-\frac{1}{\delta} \mathcal{G C}^{-1} \mathcal{D}+\mathcal{G C}^{-1} \mathcal{H}-\mathcal{H}^{T} \mathcal{C}^{-1} \mathcal{D}\right) U=F+\mathcal{G C}^{-1} \mathcal{S}^{T} F
$$

that can be shown, as in Section 2, to be positive definite provided the stabilizing parameter $\delta$ is sufficiently small. 
The pressure is recovered solving the equation

$$
\mathcal{C} P=-\frac{1}{\delta} \mathcal{D} U+\mathcal{H} U-\mathcal{S}^{T} F,
$$

which is accomplished by two wavelet transforms.

It is of primal importance to study the dependence of the discretization error on the stabilizing parameter $\delta$. To this end, we solve a Stokes problem (with nonzero right-hand side) having as exact solution $u(x, y)=v(x, y)=x(1-x) y(1-y)$ and $p(x, y)=e^{4 \cos (2 x+3 y)}$. In the construction described above, we fix $\tilde{n}_{v}=2$ and $\tilde{n}_{p}=6$ and we vary the values of $\left(J_{v}, J_{p}\right)$. Figures 1 and 2 exhibit the resulting error behavior. We can make the following observations.

(i) The minimum value of the parameter $\delta$ for which the operator is positive definite is close to 0.01 independently of the discretization. This latter value of $\delta$ always results in the best (or nearly best) approximation for the velocity.

(ii) For the discretization $\left(J_{v}=J, J_{p}=J+1\right)$, the discrete divergence free functions, i.e., the velocities $\vec{v} \in \vec{X}_{J_{v}}$ such that $(\nabla \cdot \vec{v}, q)=0, \forall q \in M_{J_{p}}$, are indeed exactly divergence free, so that the consistency error related to this constraint vanishes. Consequently, when the pressure approximation error is larger than the velocity approximation error, we expect a much lower velocity error for the discretization $\left(J_{v}=J, J_{p}=J+1\right)$, than for $\left(J_{v}=J_{p}=J\right)$ or even $\left(J_{v}=J_{p}=J+1\right)$. This is indeed the case in the numerical results displayed in Figures 1 and 2. In particular, the consistency error clearly dominates Figure 2-(E) (when compared with Figure 2-(F)), while this is not the case for Figure 2-(B).

For small $\delta$ (no stabilization effect), the discretization $\left(J_{v}=J, J_{p}=J+1\right)$ exhibits spurious modes for the pressure. They can be filtered out setting $\delta:=\delta_{p}=\infty$ in (4.2), as shown in Figure 1.

(iii) The velocity system is solved by a conjugate gradient algorithm with a diagonal type preconditioning in the velocity wavelet basis (see, e.g., [J], DK] and CM1 for numerical experiments). This ensures that the condition number of the preconditioned matrix is bounded uniformly with the scale parameter $J_{v}$.

For $\delta$ much smaller than 0.01 , the convergence is very slow although the system is positive definite. This is due to the term $-\frac{1}{\delta} \mathcal{G C}^{-1} \mathcal{D}$, which considerably deteriorates the condition number of the system that roughly behaves after preconditioning like $\frac{1}{\delta}$.

For $\delta$ larger than $0.02-0.03$, the system is no longer positive definite and the residual strongly oscillates. Thus, in order to investigate these cases, a direct solver must be used. 

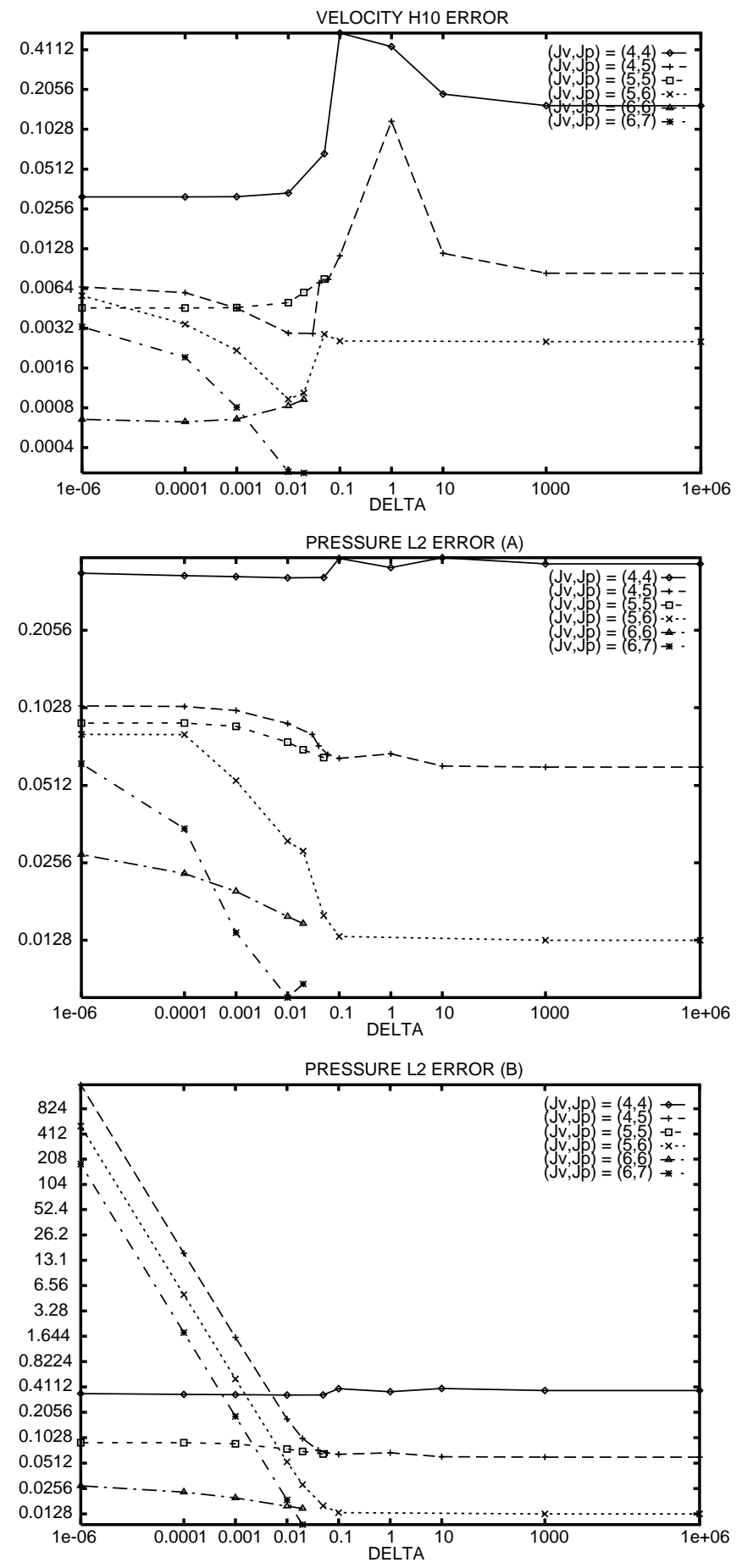

Figure 1. Dependence on $\delta$ of the velocity $H_{0}^{1}$ and pressure $L^{2}$ errors for various choices of $\left(J_{v}, J_{p}\right)\left((\mathrm{A}): \delta_{p}=\infty\right.$ for $\left(J_{v}, J_{p}\right)=$ $(J, J+1)$ and $\delta_{p}=\delta$ for $\left(J_{v}, J_{p}\right)=(J, J)$; and $(\mathrm{B}): \delta_{p}=\delta$ for all $\left.\left(J_{v}, J_{p}\right)\right)$. 


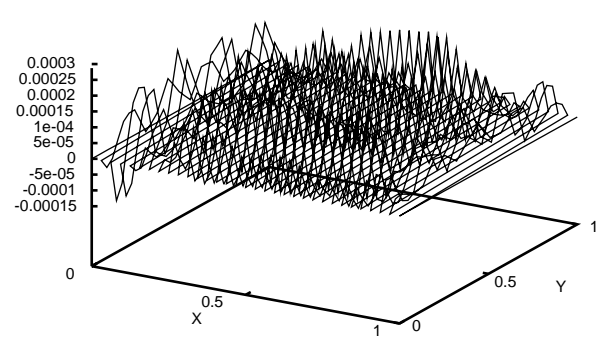

(A)

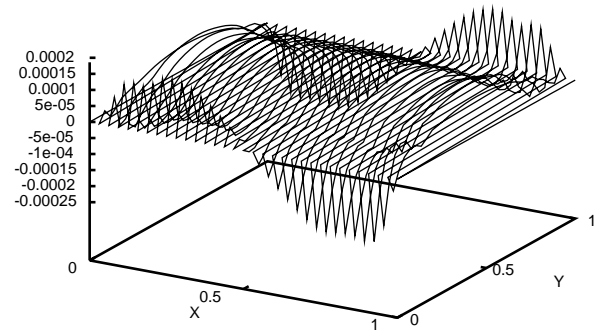

(C)

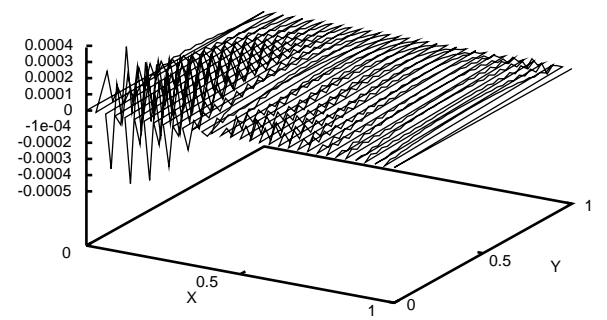

(E)

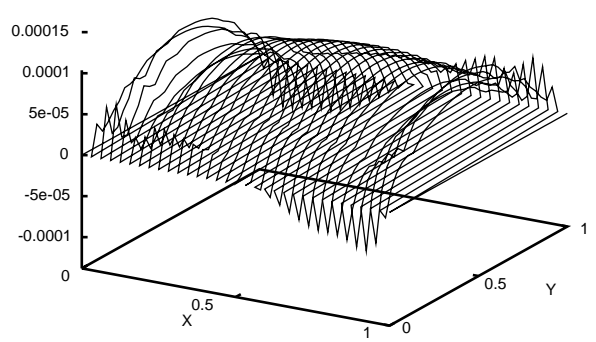

(B)

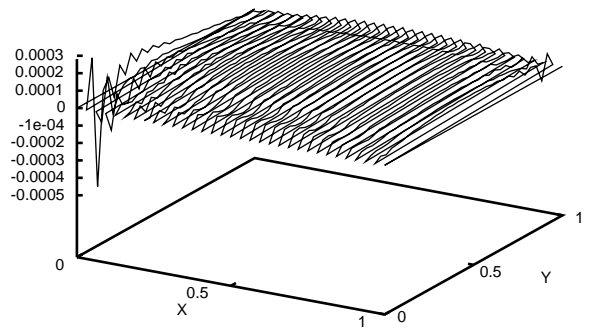

(D)

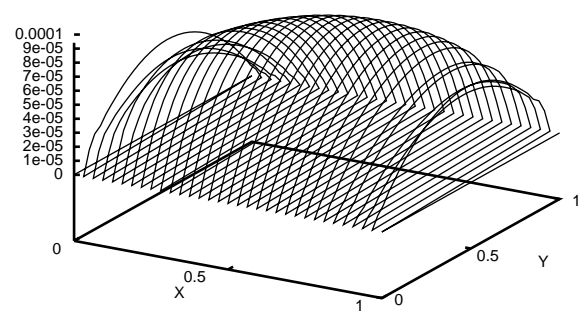

(F)

Figure 2. Error for the velocity $u,(\mathrm{~A}):\left(J_{v}, J_{p}\right)=(5,6), \delta=$ $5.10^{-2} ;(\mathrm{B}):\left(J_{v}, J_{p}\right)=(5,6), \delta=10^{-2} ;(\mathrm{C}):\left(J_{v}, J_{p}\right)=(5,6)$, $\delta=10^{-3} ;(\mathrm{D}):\left(J_{v}, J_{p}\right)=(5,6), \delta=10^{3} ;(\mathrm{E}):\left(J_{v}, J_{p}\right)=(5,5)$, $\delta=10^{-3} ;(\mathrm{F}): H_{0}^{1}$ projection error for $J_{v}=5$. 


\section{REFERENCES}

[AJP] L. Andersson, N. Hall, B. Jawerth, and G. Peters, "Wavelets on closed subsets of the real line", pp. 1-61 in Recents Advances in Wavelets Analysis (L.L.Schumaker and G. Webb, Eds.), Academic Press, San Diego, 1993. MR 94m:42072

[B] S. Bertoluzza, "Stabilization by multiscale decomposition", Appl. Math. Lett. 11 (1998), pp. 129-134. MR 99e:65168

$[\mathrm{BCT}]$ S. Bertoluzza, C. Canuto, and A. TABacco, Negative norm stabilization of convection-diffusion problems, Appl. Math. Lett., 13 (2000), pp. 121-127. CMP 2000:11

[BCU] S. Bertoluzza, C. Canuto, and K. Urban, On the adaptive computation of integrals of wavelets, Preprint n. 99-5, Dipartimento di Matematica, Politecnico di Torino; Appl. Numer. Math. 34 (2000), pp. 13-38. CMP 2000:12

[BP] J.H. Bramble, J. Pasciak, "Least-squares methods for Stokes equations based on a discrete minus one inner product", J. Comput. Appl. Math. 74 (1996), 155-173. MR 98h:73080

[BF] F. BrezzI, M. Fortin, Mixed and Hybrid Finite Element Methods, Springer-Verlag, New York, 1991. MR 92d:65187

[BFHR] F. Brezzi, L.P. Franca, T.J.R. Hughes, and A. Russo, " $b=\int g$ ", Comput. Methods Appl. Mech. Engrg. 145 (1997), 329-339. MR 98g:65086

[CTU] C. Canuto, A. Tabacco, and K. Urban, "The wavelet element method. Part I: construction and analysis", Appl. Comput. Harmon. Anal. 6 (1999), 1-52. CMP 2000:09

[Co] A. Cohen, "Wavelet Methods in Numerical Analysis", to appear in Handbook of Numerical Analysis (P.G. Ciarlet and J.L. Lions, eds.), Elsevier North Holland, Amsterdam.

[CDF] A. Cohen, I. Daubechies, and J.-C. Feauveau, "Biorthogonal bases of compactly supported wavelets", Comm. Pure Appl. Math. 45 (1992), 485-560. MR 93e:42044

[CDV] A. Cohen, I. Daubechies, and P. Vial, "Wavelets on the interval and fast wavelet transforms", Appl. Comp. Harm. Anal. 1 (1993), 54-81. MR 94m:42074

[CM1] A. Cohen, R. Masson, Wavelet Methods for Second-Order Elliptic Problems, Preconditioning, and Adaptivity, SIAM J. of Sci. Comp. 21 (1999), pp. 1006-1026. CMP 2000:12

[CM2] A. Cohen, R. MAsson, Wavelet adaptive methods for 2nd order elliptic problems. Domain decomposition and boundary conditions, Preprint No 98007, Laboratoire d'Analyse Numerique, Université Pierre et Marie Curie, 1998. Accepted in Numerishe Math.

[Da1] W. DAhmen, "Stability of multiscale transformations", J. Fourier Anal. Appl., 2 (1996), 341-361. MR 97i:46133

[Da2] W. Dahmen, "Wavelet and Multiscale Methods for Operator Equations", pp. 55-228 in Acta Numerica, Cambridge University Press, Cambridge, 1997. MR 98m:65006

[DK] W. Dahmen, A. Kunoth, "Multilevel preconditioning", Numer. Math. 63 (1992), 315344. MR 93j:65065

[DKU1] W. Dahmen, A. Kunoth, and K. Urban, "A wavelet-Galerkin method for Stokes problem", Computing 56 (1996), 259-302. MR 97g:65228

[DKU2] W. Dahmen, A. Kunoth, and K. Urban, Biorthogonal Spline Wavelets on the Interval, Stability and Moment Conditions, Appl. Comput. Harmon. Anal. 6 (1999), 132-196. MR 99m:42046

[DM] W. DAhmen, C.A. Micchelli, "Using the refinement equation for evaluating integrals of wavelets", SIAM J. Numer. Anal. 30 (1993), 507-537. MR 94c:65025

[DPS] W. Dahmen, S. Prössdorf, and R. Schneider, "Wavelet approximation methods for pseudo-differential equations II: Matrix compression and fast resolution", Adv. Comput. Math. 215 (1994), 583-620. MR 95g:65149

[DS] W. Dhhmen, R. Schneider, Composite wavelet bases for operator equations, Math. Comp. 68 (1999), 1533-1567. MR 99m:65122

[GT] S. Grivet-Talocia, A. TABAcCO, Biorthogonal wavelets on the interval with optimal support properties, Math. Models Meth. Appl. Sci., 10 (2000), pp. 441-462. CMP 2000:11

[HFB] T.J.R. Hughes, L.P. Franca, And M. Balestra, "A new finite element formulation of computational fluid dynamics. V. Circumventing the Babuska-Brezzi condition: a stable Petrov-Galerkin formulation of the Stokes problem accomodating equal-order interpolations", Comput. Methods Appl. Mech. Engrg. 59 (1986), 85-99. MR 89j:76015d

[J] S. JAFFARD, "Wavelets methods for fast resolution of elliptic problems", SIAM J. Numer. Anal. 29 (1992), 965-986. MR 93i:35042 
[L] P.G. Lemarié-Rieusset, "Analyses multirésolutions nonorthogonales, commutation entre projecteurs et dérivation et ondelettes vecteurs à divergence nulle", Rev. Mat. Iberoamer. 8 (1992), 221-236. MR 94d:42044

[Ma1] R. MASson, "Biorthogonal spline wavelets on the interval for the resolution of boundary problems", Math. Mod. Methods in Appl. Sci. 6 (1996), 749-791. MR 97k:42064

[Ma2] R. MASSON, Wavelet discretizations of the Stokes problem in velocity-pressure variables, Preprint Laboratoire d'Analyse Numerique, Université Pierre et Marie Curie, 1998.

[Me] Yves Meyer, Ondelettes et Opérateurs - Tomes 1 et 2, Hermann, Paris, 1990. MR 93i: 42002 MR 93i: 42003

[U] K. Urban, "On divergence free wavelets", Adv. in Comput. Math. 4 (1995), 51-82. MR 96e: 42035

Dipartimento di Matematica, Politecnico di Torino, Corso Duca degli Abruzzi 24, 10129 TORINO, ITALY

E-mail address: ccanuto@polito.it

Département Informatique Scientifique et Mathématiques Appliquées, Institut Français du Pétrole, BP 311, 92852 Rueil Malmaison Cedex, France

E-mail address: roland.masson@ifp.fr 